\title{
FUNGAL CORNEAL ULCERS: A PROSPECTIVE STUDY ON THE CAUSATIVE FUNGUS AND THE RESPONSE TO THE PRESENT TREATMENT PROTOCOL
}

\author{
Jisha $K^{1}$, Sreekumari P. $K^{2}$, Rajesh P. S3, K. K. Jacob ${ }^{4}$, Jayalekha $B^{5}$ \\ ${ }^{1}$ Assistant Professor, Department of Ophthalmology, Government Medical College, Kottayam. \\ ${ }^{2}$ Assistant Professor, Department of Microbiology, Government Medical College, Kottayam. \\ ${ }^{3}$ Assistant Professor, Department of Surgery, Government Medical College, Kottayam. \\ ${ }^{4}$ Additional Professor, Department of Ophthalmology, Government Medical College, Kottayam. \\ 5 Professor, Department of Microbiology, Government Medical College, Kottayam.
}

\section{ABSTRACT}

\section{BACKGROUND}

Fungal keratitis is a significant cause of ocular morbidity and unilateral blindness worldwide. The incidence varies with various geographical location and is more common in tropical countries. They are the predominant type of infective corneal ulcers in South India. Identifying the fungal spectrum in each geographical location may help to choose the initial treatment. Aim of the study was to identify spectrum of fungi, to assess the response to the standard treatment given and to find out the sensitivity and specificity of $\mathrm{KOH}$ preparation in clinically diagnosed fungal corneal ulcers in patients attending a tertiary care centre in Kerala.

\section{METHODS}

All patients who are clinically diagnosed to have fungal corneal ulcer, based on history and slit lamp findings over a period of 2 years were included in the study. All patients were subjected to corneal scraping and the material is examined by $\mathrm{KOH}$ mounting and specific culture techniques as per standard microbiological techniques. The ulcer is treated as per the standard treatment protocol followed in the institution and the response to treatment was analysed. Out of the total 81 patients, Males predominated (n=57). KOH positivity was seen in 27 cases. The fungus culture was positive in 33 cases (41\%). The sensitivity of KOH was $45.45 \%$ and specificity of $75 \%$. The most common fungus isolated was Fusarium (72.72\%) followed by Aspergillus (27.27\%). The response to the treatment was good for cases in which Fusarium (75\%) and Penicillium (100\%) were isolated. The response to the treatment was poor in cases in which the culture report was negative; $56.25 \%$ cases developed complications.

\section{CONCLUSIONS}

Among the clinically diagnosed fungal corneal ulcer cases, $40 \%$ was found to be culture positive. The $\mathrm{KOH}$ preparation has a sensitivity of $45.45 \%$ in detecting fungus in mycotic corneal ulcers, whereas the specificity is $75 \%$. Fusarium was the predominant fungus isolated. The response to the standard treatment protocol was better in fungal ulcers caused by Fusarium and Penicillium. The culture negative cases had a higher rate of complications.

\section{KEYWORDS}

Fungal Corneal Ulcer, KOH, Culture, Fungal Spectrum, Aspergillus, Fusarium.

HOW TO CITE THIS ARTICLE: Jisha K, Sreekumari PK, Rajesh PS, et al. Fungal corneal ulcers: a prospective study on the causative fungus and the response to the present treatment protocol. J. Evolution Med. Dent. Sci. 2016;5(33):1822-1826, DOI: $10.14260 /$ jemds/2016/429

\section{INTRODUCTION}

Infective corneal ulcers can be caused by bacteria, fungi, protozoa or virus. Fungal corneal ulcers are reported from different parts of the world, particularly tropical areas where it may account for more than $50 \%$ of all microbial keratitis. $1,2,3$

Fungi are opportunistic agents of infection; 70 different types of fungi are implicated as a cause for fungal corneal ulcers. $^{2}$ Fungi thrive in hot and humid environment, rich in vegetable matter and organic decay. Filamentary fungi are predominant in tropical and subtropical climates.3,4 (Fusarium and Aspergillus),

Financial or Other, Competing Interest: None.

Submission 07-03-2016, Peer Review 02-04-2016,

Acceptance 09-04-2016, Published 23-04-2016.

Corresponding Author:

Dr. Jisha $K$,

Assistant Professor

Department of Ophthalmology,

Government Medical College,

Kottayam-686008,

Kerala,

E-mail: jishakamalakshy@gmail.com

DOI: $10.14260 /$ jemds $/ 2016 / 429$ while Candida and Aspergillus appear more important in temperate and colder climates. ${ }^{5}$

Unlike western countries, the reported incidence of fungal corneal ulcers are high in our country, especially South India. This could be due to the increased humidity of our atmosphere and the fact that a considerable population work in agriculture field. ${ }^{6}$ Diagnosis and treatment of fungal corneal ulcers can be quite challenging because of the delay in seeking medical attention due to reduced symptoms in mycotic corneal ulcer and the limited antifungal agents available for ocular use and the deeper extent to which they can penetrate the corneal tissue. Isolation of the causative fungus from corneal ulcer takes weeks and thus the treatment cannot be dependent on the culture report in majority cases. Hence, the treatment given is based on clinical diagnosis supported by detecting the presence of the fungus in $\mathrm{KOH}$ mounting/Giemsa stain rather than on culture report; and a standard antifungal treatment protocol is followed in management.

Reported studies from northern part of the country isolated Aspergillus as the commonest fungus in mycotic corneal ulcers, whereas Fusarium was reported to be the commonest fungus in southern part of the country.7,8,9 This 
was in contrast to the reports from foreign countries, where candida was the commonest agent isolated. ${ }^{10}$

There are only a few reports from Kerala where Fusarium was identified as the commonest fungus causing mycotic corneal ulcer. ${ }^{11,12}$ There is a region wise variation in the predominance of fungal corneal ulcers. So the reports from outside the state cannot be considered as the basis for initiating treatment in our population. Identifying the fungal profile in mycotic corneal ulcers in a geographical location is of benefit in choosing the antifungal agents to be initiated in the event of a mycotic corneal ulcer in that area. This would be a valuable information to ophthalmologists managing corneal ulcers in rural setup, where microbiological assessment is a remote possibility.

\section{AIM OF THE STUDY}

To identify spectrum of fungi in fungal corneal ulcers in patients attending Government Medical College, Kottayam.

To assess the response of the specific fungus to the standard treatment given to patients with fungal corneal ulcer.

To find out the sensitivity of $\mathrm{KOH}$ preparation in detecting fungal hyphae in clinically diagnosed fungal corneal ulcers.

\section{MATERIALS AND METHODS}

It was a prospective study conducted at the Department of Ophthalmology, Government Medical College, Kottayam, Kerala, for a period of 2 years from September 2011 to August 2013. All patients with a clinical diagnosis of fungal corneal ulcer based on the history and the slit lamp examination findings were included in the study after obtaining a written consent. The clinical features considered in making the diagnosis of fungal corneal ulcer were history of injury with a vegetative matter, signs out of proportion to the symptoms, firm dry elevated slough, hyphate lines extending the ulcer edge into the normal cornea, multifocal granular or feathery grey white satellite stromal infiltrates, immune ring, endothelial plaque and immobile hypopyon.1,2 Institutional Ethical Committee approval was obtained for conducting the study. Children who are not cooperative for taking the corneal scrapings were excluded from the study; 81 patients who were clinically diagnosed to have fungal corneal ulcer based on the findings described above were included in the study.

After a detailed ocular examination, corneal scrapings were collected using a sterile Bard-Parker blade no. 5 under strict aseptic conditions from each ulcer by an ophthalmologist after instillation of proparacaine $10.5 \%$ eye drop). The procedure was performed under magnification of slit-lamp.

The scraping material first obtained from the leading edge and base of each ulcer was initially directly inoculated onto the surface of solid media Sabouraud dextrose agar and the next scraping was inoculated into blood agar.

The materials obtained by the subsequent scrapings were spread onto labelled slides in a thin, even manner for $10 \%$ potassium hydroxide $(\mathrm{KOH})$ wet mount and Gram staining. The smear is examined under high power of microscope. Meticulous care was taken in the collection of material and transferring it to the appropriate culture media aseptically. The Blood Agar is incubated at 38 degrees Celsius for 48 hours to assess for any growth the inoculated media Sabouraud dextrose agar was incubated aerobically in
Biological Oxygen Demand Incubator at 22 degrees Celsius. It is inspected for growth daily till 3 weeks. If growth is seen, the organism is identified by Lactophenol cotton blue staining and slide culture will be done to confirm. If no growth is seen after 3 weeks, it is discarded and reported as culture negative. All laboratory methods followed standard protocols. ${ }^{3}$

The present protocol for treatment is that the initial treatment will be started based on the clinical picture and smear report. Topical 5\% Natamycin eye drop applied hourly for filamentous fungus. Topical Azole group of antibiotic for yeasts. Systemic antifungals will be added if the ulcer is large/deep/hypopyon is present. Tab Itraconazole $100 \mathrm{mg} \mathrm{BD}$ given for three weeks is the systemic antifungal agent administered. For a proved fungal ulcer, which is not responding to the above regimen, second preferred drug is topical Amphotericin B and Voriconazole. Ulcers which are getting worse even to the above treatment regimen will be taken for therapeutic keratoplasty. Large perforated total corneal ulcers, which have responded to treatment will be subjected to conjunctival hood flapping. Complicated cases where there was panophthalmitis, evisceration was the treatment option preferred.

The Response to $R x$ will be Analysed based on the Following Parameters

1. Improvement/worsening is noted based on changes in symptom size and depth of ulcer, margins, surrounding cornea $\mathrm{AC}$ reaction.

2. The final result is recorded as healed or not healed.

3. Complications-Total ulceration, Thinning of cornea Descemetocele, Perforation, Endophthalmitis or panophthalmitis.

4. Need for surgical intervention like Therapeutic corneal graft, Conjunctival hood flap or Evisceration will be noted.

The data collected from 81 patients using a case report form. Demographic profile, clinical findings, $\mathrm{KOH}$ preparation report, culture report, treatment given and response to treatment were noted down in the case report form.

\section{RESULTS}

81 patients with a clinical diagnosis of fungal corneal ulcer were included in the study.

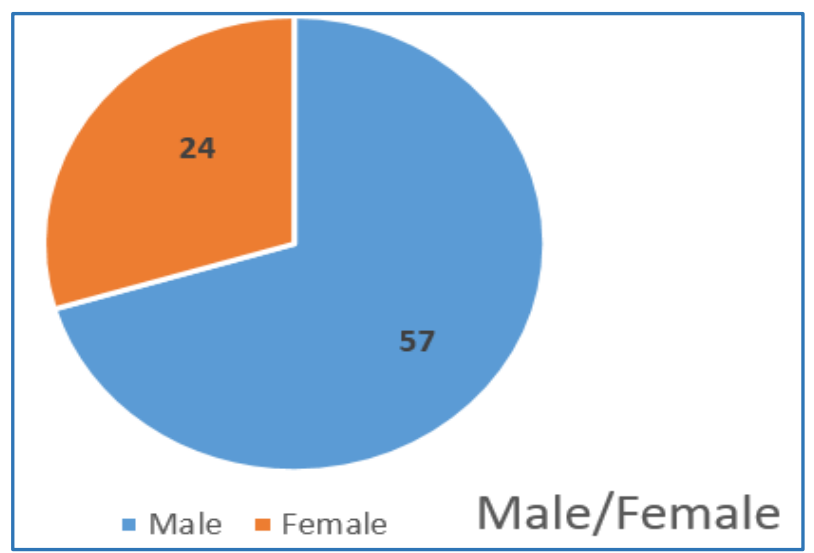

Fig. 1: Distribution of Study Subjects Based on Gender

Males predominated in the study population than females. 


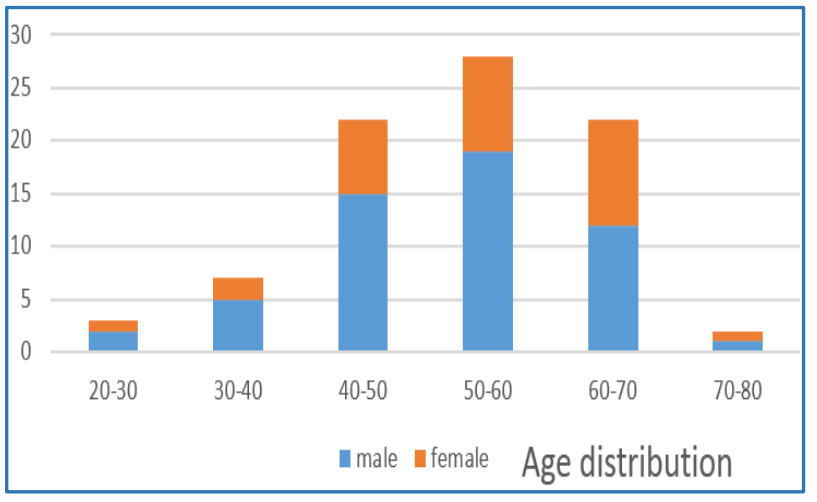

Fig. 2: Age Group Distribution of Study Population

Majority of the patients belonged to the age group of 5060 years (34.5\%) followed by the age group 40-50 (25.9\%).

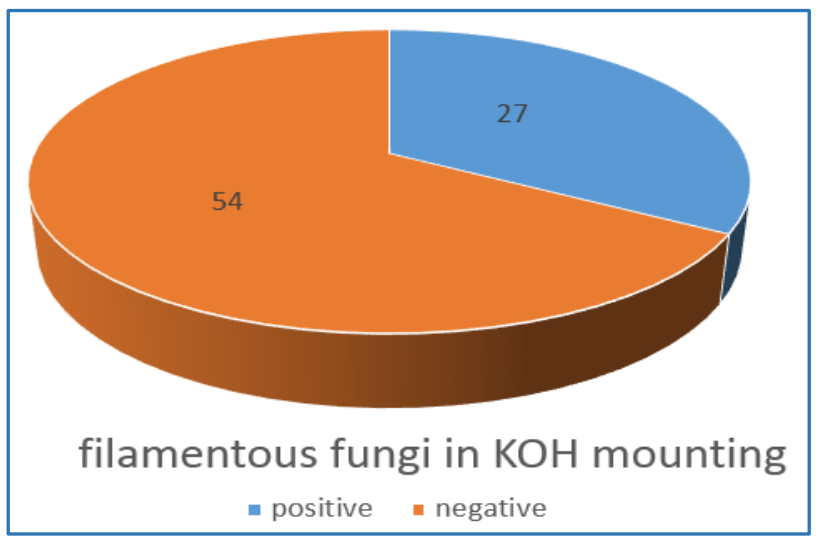

Fig. 3: Result of КОН Preparation

KOH preparation of the corneal scraping revealed that only 27 out of 81 cases had the presence of filamentous fungi (33\%). Rest of the cases did not reveal the presence of any fungi.

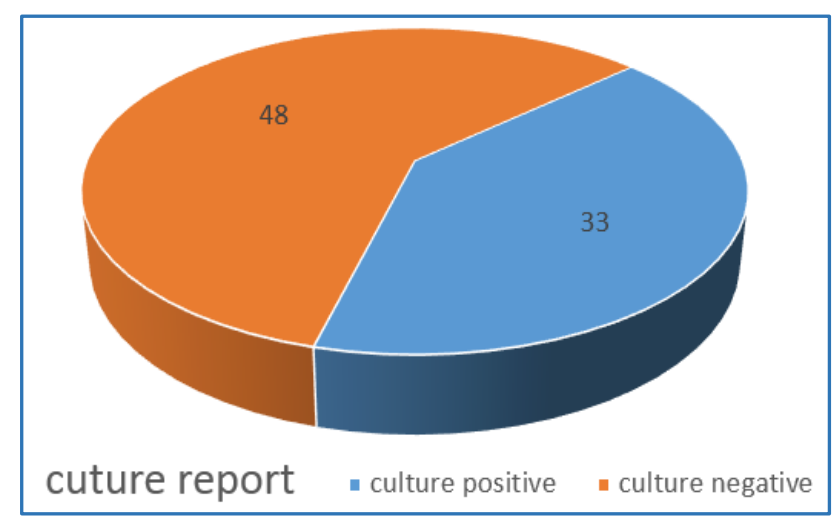

Fig. 4: Results of Fungal Culture

Culture results showed the presence of fungal growth in 33 cases (41\%) whereas 48 cases (59\%) were culture negative for fungi.

\begin{tabular}{|l|c|c|}
\hline & $\begin{array}{c}\text { KOH Positive } \\
(\mathbf{n = 2 7})\end{array}$ & $\begin{array}{c}\text { KOH Negative } \\
(\mathbf{n = 5 4 )}\end{array}$ \\
\hline Culture positive $(\mathrm{n}=33)$ & 15 & 18 \\
\hline Culture negative (n=48) & 12 & 36 \\
\hline \multicolumn{2}{|c|}{ Table 1: Comparison of KOH Result with Culture Report } \\
\hline
\end{tabular}

The $\mathrm{KOH}$ results were compared and analysed with the results of the culture. It was found that out of the $27 \mathrm{KOH}$ positive cases fungal growth by culture was yielded only in 15 cases $(55.5 \%)$ whereas the $44.5 \%$ cases $(n=12)$ could not isolate any fungi in spite of having a KOH Positivity. Out of 54 $\mathrm{KOH}$ negative cases 18 cases revealed the growth of fungi (33\%) and remaining were confirming the negativity of $\mathrm{KOH}$ by culture $(67 \%)$. The sensitivity of detection of a fungus by $\mathrm{KOH}$ preparation was found to be $45.45 \%$ and the specificity was $75 \%$.

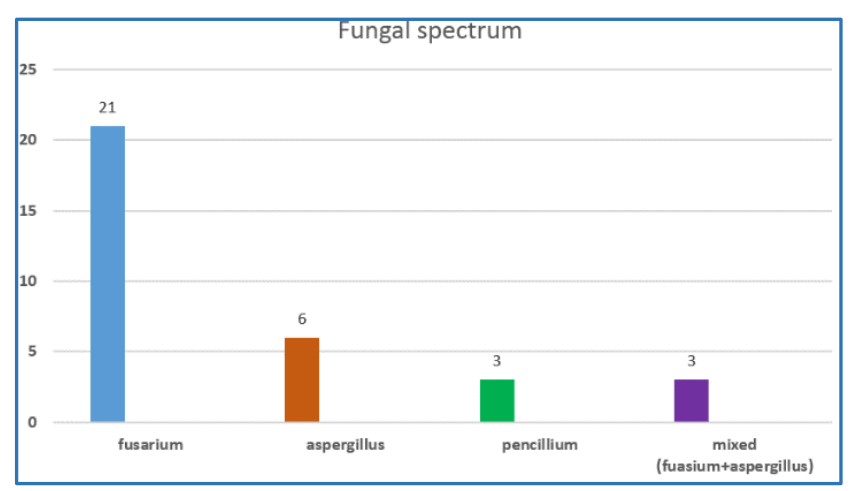

Fig. 5: Distribution of Fungal Spectrum in Culture Positive Cases

The culture positive cases isolated Fusarium spp. in 21 cases, Aspergillus in 6 cases, Penicillium in 3 cases, mixed infections with Penicillium and Aspergillus in 3 cases. Fusarium was found to be the predominant fungi isolated from the cases studied $72.72 \%(n=24)$ followed by Aspergillus fumigatus $27.27 \%(n=9)$ (Including the mixed infection).

The response to the standard treatment protocol was analysed for all the cases included in the study. This revealed that among the culture positive cases, $73 \%(n=24)$ responded well to the treatment and healed during follow-up without any complication; whereas $27 \%(\mathrm{n}=9)$ cases had complications met with which required either conjunctival hood flapping or keratoplasty. Among the Fusarium isolated cases (Including mixed infections) $75 \%$ cases $(n=18)$ showed good response to the standard protocol and healed well, whereas $25 \%(n=6)$ cases developed complications. Of this and 3 cases worsened rapidly to near total corneal ulcer and 3 cases perforated. All cases in which Penicillium was isolated $(n=3)$ showed good response to treatment and healed (100\%). Among the cases in which Aspergillus was isolated (Including mixed infections), $66 \%(n=6)$ of cases healed with treatment; 3 cases required therapeutic keratoplasty. One case worsened in spite of therapeutic keratoplasty and required conjunctival hood flapping.

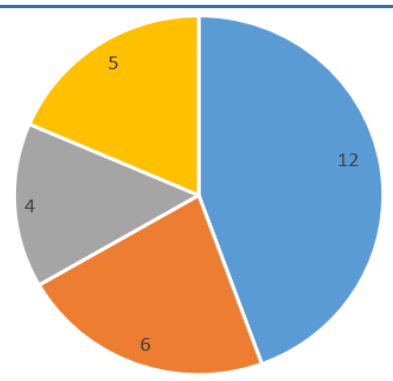

complications in culture negative fungal corneal ulcer

- perforation $=$ Descematocele $\quad$ - total corneal ulcer $\quad$ near total corneal ulcer

Fig. 6: Distribution of the Rate of Complications in Culture Negative Cases 
Among the culture negative cases, $43.75 \%(n=21)$ healed with treatment; $56.25 \%(\mathrm{n}=27)$ had met with complications of which 12 cases had perforation, 6 cases had descemetocele, 4 cases had total corneal ulceration, 5 cases had near total corneal ulcer with large hypopyon. Of these 12 cases had undergone therapeutic keratoplasty and the success rate was only $50 \%$.

\section{DISCUSSION}

Mycotic keratitis is the second most common reason for corneal blindness in developing countries. ${ }^{13}$ Various published reports indicate that mycotic keratitis account for $6 \%$ to $50 \%$ of all cases of ulcerative keratitis. ${ }^{14,15,16}$ Diagnosis and treatment of fungal corneal ulcers is one of the difficult tasks encountered by an ophthalmologist.

This study found that fungal corneal ulcer was more common in males $(n=57)$ than females $(n=24)$. This was similar to majority of the reported cases where the male population predominated in having fungal corneal ulcer. $3,7,8,11$ A study done in Oman reported that $59.37 \%$ were males. ${ }^{17}$ and another study in northern India also reported that males are affected more commonly (68\%) than females. ${ }^{13}$ However, there is a report from Philadelphia which reported a slightly more occurrence of fungal ulcer in females (58.3\%). ${ }^{5}$

Direct microscopic detection of fungal elements in corneal scrapings is a most valuable and rapid diagnostic tool in the diagnosis of fungal corneal ulcers. ${ }^{18,19,20}$ Of the various methods available for the microscopic detection of fungus, $\mathrm{KOH}$ preparation is one of the oldest methods. ${ }^{20}$ Various studies revealed the utility of $\mathrm{KOH}$ preparation in fungal corneal ulcers. ${ }^{8,18,20,21,22}$ The sensitivity of $\mathrm{KOH}$ preparation has been found to be variable ranging between $33-94 \%$ as reported by various studies. The sensitivity and specificity of $\mathrm{KOH}$ preparation in our study was $45.45 \%$ and $75 \%$ respectively. This was much lower than that reported by Jayabar Bharathi et al ${ }^{18}$, where the sensitivity was $99.23 \%$. In another study done in Iran, it was found to be $71.4 \%$ and Rajpal Singh et al ${ }^{8}$ reported a sensitivity of $88.64 \%$ and specificity of $98 \%$.

The sensitivity and specificity of $\mathrm{KOH}$ mounting in the detection of fungi in clinically diagnosed fungal corneal ulcer was $45 \%$ and $75 \%$ respectively. This was much different from other reported series.20,21 In a study by Bandyopadhyay $\mathrm{S}$ et al, the sensitivity was $98.3 \%$ and specificity was $98.5 \%$. Fungal Hyphae were seen by wet mount preparation in $80.9 \%$ cases and culture growth present in $88 \%$ cases as reported by $\mathrm{V}$ Sharma et al $^{20}$

The fungal culture positivity was $41 \%$ in our study. Bandyopadhyay et al ${ }^{21}$ reported that $72.9 \%$ corneal ulcers were culture positive. This figure agrees with previous studies in West Bengal, Chennai and Ghana.6,21 Studies done in tropical countries like Bangladesh and Nepal reported the incidence from $17 \%$ to $40 \% .^{16}$

More than 70 species of fungi have been reported to cause mycotic corneal ulcers. The fungal spectrum isolated in mycotic ulcer showed a difference in the predominant species depending on the geographical location. Our study reported Fusarium as the commonest organism. This was similar to other studies from Kerala itself, which showed similar results.11,12 In a study by Gopinath et al7, the most common isolate was Aspergillus followed by Fusarium. Aspergillus was the predominant isolate in many other studies.8,9,23,24 In a published study, Rajpal Singh et al ${ }^{8}$ reported that $59.09 \%$ was Aspergillus and $15.9 \%$ was Fusarium. However, Fusarium was found to be the common in some other studies done in South India. ${ }^{4,24}$ and some foreign countries like Oman Paraguay, Hong Kong and Singapore.17,19 Candida was the most common isolate identified in a study from USA. ${ }^{5}$ These differences in the fungal isolates could be attributed to the different climatic conditions, which favour the growth.

As with all corneal infections, accurate identification of microbe and targeted therapy can alleviate the complications.

\section{CONCLUSIONS}

1. Among the clinically diagnosed fungal corneal ulcer cases, $40 \%$ was found to be culture positive.

2. The sensitivity of $\mathrm{KOH}$ preparation has a sensitivity of $45.45 \%$ in detecting fungus in mycotic corneal ulcers, whereas the specificity is $75 \%$.

3. Fusarium was the predominant fungus isolated from the clinically diagnosed fungal corneal ulcers.

4. The response to the standard treatment protocol was better in fungal ulcers caused by Fusarium and Penicillium.

5. The culture negative cases had a higher rate of complications, probably due to the unidentified organisms.

\section{REFERENCES}

1. Thomas PA. Current perspective on ophthalmic mycosis. Clin Microbiol Rev 2003;16(4):730-97.

2. Thomas PA. Fungal infections of the cornea. Eye 2003;17(8):852-62.

3. Srinivasana M, Christine A Gonzalesb, Celine Georgea. Epidemiology and aetiological diagnosis of corneal ulceration in Madurai, south India. Br J Ophthalmol 1997;81(11):965-71.

4. Srinivasan M. Fungal keratitis. Current Opinion in Ophthalmology 2004;15(4):321-7.

5. Tanure MA, Cohen EJ, Sudesh S, et al. Spectrum of fungal keratitis at wills eye hospital, philadelphia, pennsylvania. Cornea 2000;19(3):307-12.

6. Leck AK, Thomas PA, Hagan M, et al. Aetiology of suppurative corneal ulcers in ghana and south India, and epidemiology of fungal keratitis. Br $\mathrm{J}$ Ophthalmol 2002;86(11):1211-5.

7. Gopinathan $U$, Garg $P$, Fernandes $M$, et al. The epidemiological features and laboratory results of fungal keratitis: a 10-year review at a referral eye care center in south India. Cornea 2002;21(6):555-9.

8. Rajpal Singh Punia, Reetu Kundu, Jagdish Chander, et al. Spectrum of fungal keratitis: clinicopathologic study of 44 cases. Int J Ophthalmol 2014;7(1):114-7.

9. Jagdish Chander, Nidhi Singla, Nalini Agnihotri, et al. Keratomycosis in and around chandigarh: a five-year study from a north Indian tertiary care hospital. Indian J Pathol Microbiol 2008;51(2):304-6.

10. Ritterband DC, Seedor JA, Shah MK, et al. Fungal keratitis at the new york eye and ear infirmary. Cornea 2006;25(3):264-7.

11. Raju KV, Vijayalakshmi MS, Lakshmi J. Clinical study of fungal corneal ulcer. Kerala Journal of Ophthalmology 2008; Vol XX(2). 
12. Geethakumari PV, Remya R, Girijadevi MS, et al. Bacterial keratitis and fungal keratitis in south kerala: a comparative study. Kerala Journal of ophthalmology 2011; Vol XXIII(1).

13. Chowdhary A, Singh K. Spectrum of fungal keratitis in north India. Cornea 2005;24(1):8-15.

14. Rosa RH, Miller D, Alfonso EC. The changing spectrum of fungal keratitis in south florida. Ophthalmology 1994;101(6):1005-13.

15. Upadhyay MP, Karmacharya PC, Koirala S, et al. Epidemiologic characteristics, predisposing factors, and aetiologic diagnosis of corneal ulceration in Nepal. Am J Ophthalmol 1991;111(1):92-9.

16. Katz NN, Wadud SA, Ayazuddin M. Corneal ulcer disease in Bangladesh. Ann Ophthalmol 1983;15(9):834-6.

17. Thara Idiculla, George Zachariah, Keshav BR, et al. A retrospective study of fungal corneal ulcers in the south sharqiyah region in oman. Sultan Qaboos Univ Med J 2009;9(1):59-62.

18. Jayahar Bharathi M, Ramakrishnan R, Vasu S, et al. Epidemiological characteristics and laboratory diagnosis of fungal keratitis a three-year study. Indian J Ophthalmol 2003;51(4):315-21.
19. Gugnan HC, Talwar RS, Njoku-Obi AN, et al. Mycotic keratitis in nigeria a study of 21 cases. $\mathrm{Br} \mathrm{J}$ Ophthalmol 1976;60(9):607-13.

20. Sharma S, Silverberg M, Mehta P, et al. Early diagnosis of mycotic keratitis: predictive value of potassium hydroxide preparation. Indian J Ophthalmol 1998;46(1):31-5.

21. Bandyopadhyay S, Das D, Mondal KK, et al. Epidemiology and laboratory diagnosis of fungal corneal ulcer in the sundarban region of West Bengal, eastern India. Nepal J Ophthalmol 2012;4(1):29-36.

22. Bharathi MJ, Ramakrishnan R, Meenakshi $\mathrm{R}$, et al. Microbiological diagnosis of infective keratitis: comparative evaluation of direct microscopy and culture results. Br J Ophthalmol 2006;90(10):1271-6.

23. Bashir G, Shah A, Thokar MA, et al. Bacterial and fungal profile of corneal ulcers-a prospective study. Indian J Pathol Microbiol 2005;48(2):273-7.

24. Tilak R, Singh A, Maurya OP, et al. Mycotic keratitis in India: a five-year retrospective study. J Infect Dev Ctries 2010;4(3):171-4. 